\title{
The genus Actinoallomurus and some of its metabolites
}

\author{
Roberta Pozzi ${ }^{1,2}$, Matteo Simone ${ }^{2,3}$, Carlo Mazzetti ${ }^{2,3}$, Sonia Maffioli ${ }^{2}$, Paolo Monciardini ${ }^{1,4}$, Linda Cavaletti ${ }^{4}$, \\ Ruggiero Bamonte ${ }^{4}$, Margherita Sosio ${ }^{1,2}$ and Stefano Donadio ${ }^{1,2}$
}

In the search for novel antibiotics, natural products continue to represent a valid source of bioactive molecules. During a program aimed at identifying previously unreported taxa of actinomycetes as potential source of novel compounds, we isolated hundreds of different representatives of a new group, initially designated as 'Alpha' and independently described as Actinoallomurus. We report on a PCR-specific method for the detection of this taxon, on appropriate growth conditions and on a pilot-screening program on $\mathbf{7 8}$ strains. The strains produce antibacterial or antifungal compounds at a relatively high frequency. Four strains were characterized in further detail: one produced the aromatic polyketide benanomicin B and its dexylosyl derivative; a second strain produced $\boldsymbol{N}$-butylbenzenesulfonamide; a third strain was an efficient converter of soymeal isoflavonoids from soymeal constituents; and a fourth strain produced several coumermycin-related aminocoumarins, with coumermycin A2 as the major peak, and with some new congeners as minor components of the complex. These data suggest that Actinoallomurus strains possess several pathways for secondary metabolism and represent an attractive source in the search for novel antibiotics.

The Journal of Antibiotics (2011) 64, 133-139; doi:10.1038/ja.2010.149; published online 1 December 2010

Keywords: actinomycetes; screening; secondary metabolites

\section{INTRODUCTION}

New antibiotics are urgently needed because of the increasing spread of resistant pathogens and the evolution of new diseases. As microbial genomes, synthetic libraries and high throughput screening have so far failed to produce a significant number of developmental candidates in the antibacterial field, it is generally acknowledged that nature will continue to be a significant source of novel bioactive compounds, ${ }^{1}$ as confirmed by a recent survey of antibiotics under clinical development. ${ }^{2}$ However, in a background of several thousands of known microbial metabolites, it is necessary to introduce new strategies for accessing microbial strains to increase the probability of finding novel compounds. Chemical screening ${ }^{3}$ and, more recently, genome mining ${ }^{4}$ are alternatives to the traditional bioassay-guided screening. In the latter case, the probability of finding new compounds may be increased by the use of novel assays, of novel microorganisms or by the mere size of the library to be screened. ${ }^{5}$ In any case, dedicated efforts are providing novel metabolites. ${ }^{6}$

In the past years, our attention has been drawn to previously uncultured actinomycetes, under the assumption that strains belonging to novel taxa, which are however phylogenetically related to prolific producers of secondary metabolites, have a high chance of possessing diverse pathways in secondary metabolism and of affording novel compounds. ${ }^{7}$ A 16 S-guided isolation program led to the identification of several new taxa, ${ }^{7}$ three of which have been subsequently formally described. ${ }^{8-10}$ During that program we were also able to isolate hundreds of different strains provisionally designated as 'Alpha', whose phylogenetic position suggested a new taxon within the Actinobacteria. ${ }^{7}$ Independently, phylogenetically related strains have been assigned to the recently described genus Actinoallomurus. ${ }^{11}$

We present here a characterization of 'Alpha' strains present in our collections, in terms of their potential to produce secondary metabolites. The results from a small pilot study suggest that the genus Actinoallomurus has the potential to produce metabolites arising from different biosynthetic pathways.

\section{RESULTS}

In the course of isolation programs, aimed at identifying actinomycetes belonging to new taxa, ${ }^{7}$ we isolated from soil samples several actinomycete strains, with branching aerial hyphae that, as observed by light microscopy on agar plates, differentiated into single spore chains along the aerial mycelium, reminiscent of some Actinomadura strains. Most of the strains were able to grow in the $30-37^{\circ} \mathrm{C}$ temperature range at an optimal $\mathrm{pH}$ of 5.5. Phylogenetic analysis of selected strains indicated they belong to the recently described genus Actinoallomurus within the Thermomonosporaceae. ${ }^{11}$ Furthermore, on $16 \mathrm{~S}$ rRNA analysis, we were able to subdivide 115 strains in 32 'Alp'

\footnotetext{
${ }^{1}$ KtedoGen Srl, Milano, Italy; ${ }^{2}$ NAICONS Scrl, Milano, Italy; ${ }^{3}$ ITB-CNR, Segrate, Italy and ${ }^{4}$ Vicuron Pharmaceuticals, Gerenzano, Italy Correspondence: Dr S Donadio, NAICONS Scrl, Via Fantoli 16/15, 20138 Milano, Italy. E-mail: sdonadio@naicons.com 
phylotypes (Figure 1), indicating a substantial diversity within this genus.

For the rapid identification of novel isolates, we developed an Actinoallomurus-specific PCR method (see Methods). When we applied this method to soil samples, about $25 \%$ of soil DNA preparations yielded a band of the expected size, ${ }^{7}$ suggesting that Actinoallomurus strains may be quite cosmopolitan. Consistently, strains with an alpha-type morphology were isolated from many different soils, and the PCR method proved an effective tool for rapidly assigning most of them to Actinoallomurus.

The Thermomonosporaceae family currently comprises five genera but, to our knowledge, only strains belonging to the genus Actinomadura have been reported to produce secondary metabolites. To evaluate the ability of Actinoallomurus strains to produce bioactive compounds, we identified suitable growth conditions (see Methods). Examples of HPLC profiles are reported in Figure 2: different strains yielded different profiles, suggesting production of different secondary metabolites. Next, a pilot-screening program was carried out on
78 different strains belonging to 26 different phylotypes. About $30 \%$ of the tested strains showed at least an antimicrobial activity in our conditions (Table 1). These results prompted us to further investigate these strains and identify the bioactive metabolites produced. Here, we report the metabolites identified from four strains belonging to different phylotypes. The data are summarized in Table 2.

\section{Strain K15}

Strain K15 produced an antifungal activity and a diffusible red pigment. In all tested media, it showed good growth, and production of the red pigment and of the antifungal activity, both of which could be efficiently recovered from the mycelium by $\mathrm{MeOH}$ extraction. HPLC fractionation of the MeOH extract, followed by LC-MS of the active fractions, led to the identification of two peaks eluting at 14.5 and 16.0 mins, containing the major antifungal activity (Figure 3 ). These peaks exhibited UV-VIS maxima at $500 \mathrm{~nm}$ and showed $\left[\mathrm{MH}^{+}\right]$ of 827 and $696 \mathrm{~m} / \mathrm{z}$, respectively. Searches in the ABL database identified the antifungal compounds benanomicin B and its dexylosyl

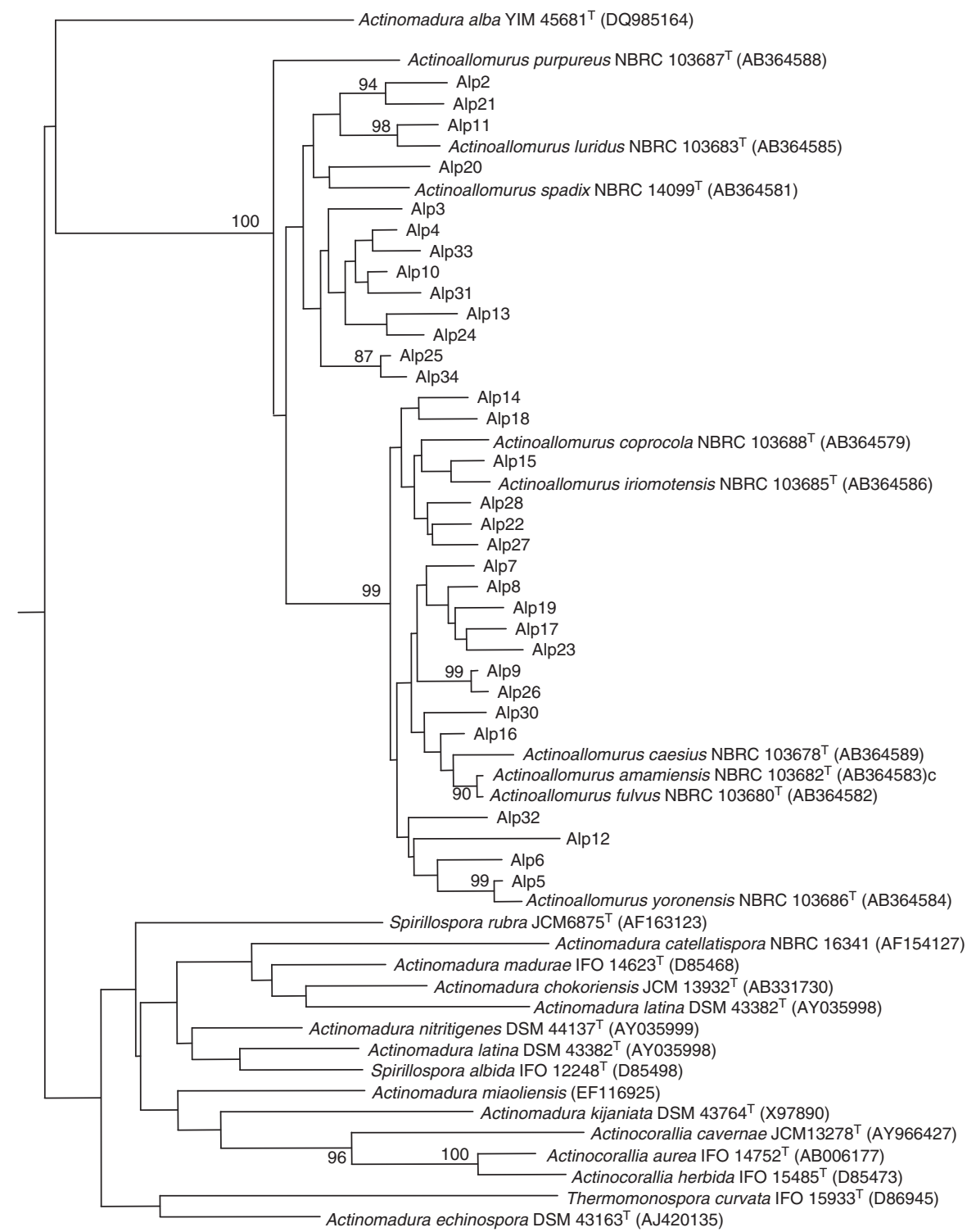

Figure 1 The 'Alp' phylotypes within Actinoallomurus. Phylogenetic tree of one representative from each Alp phylotype, nine Actinoallomurus species and selected representatives of other Thermomonosporaceae genera. 


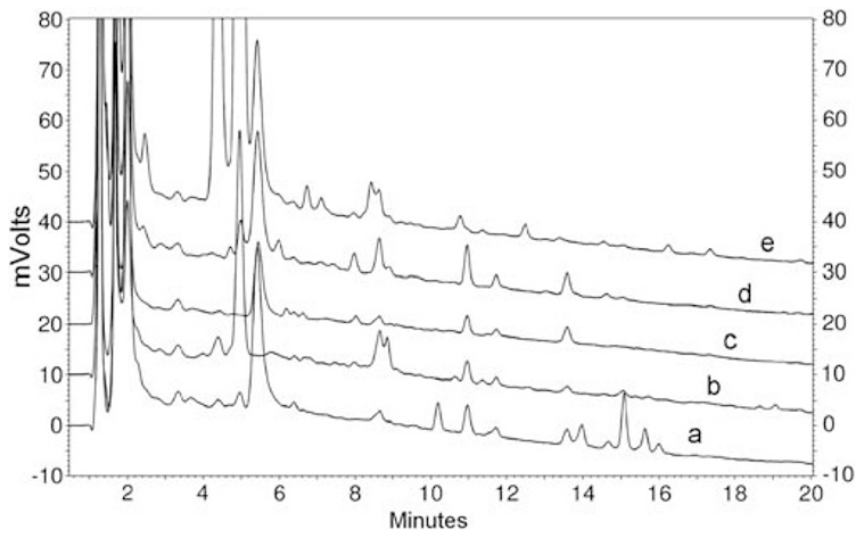

Figure 2 HPLC profiles of selected 'Alpha' strains. Strains were grown in AF5.5 for 9 days and $\mathrm{MeOH}$ extracts from entire culture were analyzed as described under Methods, with detector set at $230 \mathrm{~nm}$. Strains were as follows: a, K15; b, K79; c, K247; d, K275; and e, K277.

Table 1 Summary of screening results

\begin{tabular}{lccc}
\hline Test strain & Strains & Positives & Confirmed as Alp \\
\hline S. aureus & 78 & $22^{\mathrm{b}}$ & $21^{\mathrm{b}}$ \\
E. coli & 78 & $6^{\mathrm{b}}$ & $6^{\mathrm{b}}$ \\
C. albicans & 78 & 4 & 3 \\
\hline
\end{tabular}

Abbreviation: Alp, Alpha.

aBy $16 \mathrm{~S}$ rRNA gene sequencing and/or by the specific PCR method.

bFive strains showed activity on both $S$. aureus and $E$. coli.

derivative, respectively, ${ }^{12}$ as likely candidates for the metabolites produced by strain K15. This was confirmed on a larger preparation of the 14.5-min fraction from the NMR signals at 6.9-7.27 and 3.24.75 p.p.m., expected from aromatic and sugar protons, respectively, and by observing MS/MS fragmentations of $\mathrm{m} / \mathrm{z}$ values of 695,550 and 461, expected from the loss of the first and the second sugar and the alanine residue, respectively (data not shown).

\section{Strain K216}

Strain K216, selected for its anti-Streptomyces aureus activity, produced on agar plates, a cell-associated violet pigment turning dark green with age. This activity could be recovered from both the mycelium and the cleared broth. Ethyl acetate extraction of the cleared fermentation broth, HPLC fractionation and LC-MS analyses led to the identification of one peak eluting at $15.7 \mathrm{~min}$ containing the major antibiotic activity (Figure 4). One of the molecules present in this fraction showed a $[\mathrm{M}+\mathrm{H}]^{+}$of $214 \mathrm{~m} / \mathrm{z}$. Search in the ABL database suggested $\mathrm{N}$-butylbenzenesulphonamide ${ }^{13}$ as the likely compound. NMR analysis confirmed this structure by comparison with the literature data.

\section{Strain K241}

K241 sporulates well on agar plates, in which it produces an agardiffusible black pigment. The strain produced an antistaphylococcal activity, which was observed in the mycelium from INA5-15 cultures, despite a relatively poor growth in this medium. LC-MS analysis identified $[\mathrm{M}+\mathrm{H}]^{+}$peaks with $\mathrm{m} / z$ values of 271,285 and 255 , which matched the isoflavonoids genistein, 7-O-methyl-genistein and daidzein, respectively. These compounds, which show antibacterial activity as well as different pharmacological effects in animal cells, ${ }^{14}$ have been reported before from actinomycetes, ${ }^{15}$ although it has been suggested that these metabolites derive from microbial hydrolysis of glycosidic plant isoflavonoids present in the culture medium rather than from
Table 2 Summary of analyzed strains

\begin{tabular}{llll}
\hline Alp strain & Phylotype & Original bioactivity & Identified metabolite(s) \\
\hline K15 & Alp13 & C. albicans & Benanomicin B \\
K216 & Alp25 & S. aureus & N-Butylbenzenesulphonamide \\
K241 & Alp16 & S. aureus & Genistein and related compounds \\
K275 & Alp18 & S. aureus & Coumermycins \\
\hline
\end{tabular}

Abbreviation: Alp, Alpha.

de novo biosynthesis. ${ }^{16}$ Consistently, HPLC analyses of organic extracts from strain K241 grown in INA5 medium containing $5 \mathrm{gl}^{-1}$ instead of $15 \mathrm{gl}^{-1}$ of soybean flour showed a corresponding decrease in the height of the genistein, 7-O-methylgenistein and daidzein peaks, which were absent altogether when soybean flour was omitted from INA5 or in a soybean flour-free medium such as RARE3 medium (Figure 5). These data suggest that strain K241 produces one or more enzymes able to deglycosylate plant isoflavonoids. Interestingly, irrespective of the presence of isoflavonoids, methanolic extracts still retained an activity against $S$. aureus, which still awaits identification.

\section{Strain K275}

Colonies of strain K275 progressed from ocher to violet on agar plates. From a mycelium methanolic extract, the HPLC fractions, containing antistaphylococcal activity, matched in their UV and MS properties (Figure 6) with many congeners of the coumermycin complex. ${ }^{17} \mathrm{NMR}$ analysis of the most active fraction confirmed the presence of coumermycin A2 $\left(m / z \quad 1082[\mathrm{M}+\mathrm{H}]^{+}\right)$, whereas we inferred the presence of coumermycins D1, C and D $(\mathrm{m} / \mathrm{z}$ values of 1096,989 and $908[\mathrm{M}+\mathrm{H}]^{+}$, respectively) by MS and MS/MS fragmentation. As minor components of the complex, we also identified potentially new coumermycin congeners showing $\mathrm{m} / z$ values of 1068 and 975 $[\mathrm{M}+\mathrm{H}]^{+}$, with fragmentation patterns similar to coumermycin $\mathrm{A} 2$ and $\mathrm{C}$, respectively. The small amounts of these congeners, amid large amounts of coumermycin A2, D1, C and D, prevented us from carrying out their structural characterization.

\section{DISCUSSION}

Strains belonging to the genus Actinoallomurus seem to be present in different environments, as indicated by our isolation efforts and by screening soil DNA with our PCR method, as well as by their retrieval from different sources, including soils, plants and dung, by other groups. ${ }^{11,18-20}$ Furthermore, our limited phylogenetic analysis indicates a discrete level of genotypic diversity. Thus, this genus appears to fulfill one requisite for being a valid source of bioactive compounds: the possibility to access a significant number of strains with a reasonable degree of diversity. Here, we have also started to address the bioactive metabolites produced by Actinoallomurus strains.

Despite the use of a limited number of culturing conditions, our results indicate that about $30 \%$ of the screened strains produce an antimicrobial activity against three test strains, a fraction that favorably compares with other, well-established genera of filamentous actinomycetes. From three different Actinoallomurus strains, we identified three unrelated compounds belonging to different chemical classes: benanomicin, coumermycin and $N$-butylbenzensulphonamide. Benanomicin- and coumermycin-related compounds have been reported as the products of Actinomadura or Streptomyces strains. $\mathrm{N}$-butylbenzensulphonamide, an antifungal compound active against fungal plant pathogens, has been reported from a greenhouseassociated Pseudomonas strain ${ }^{13}$ and, very recently, from a terrestrial 

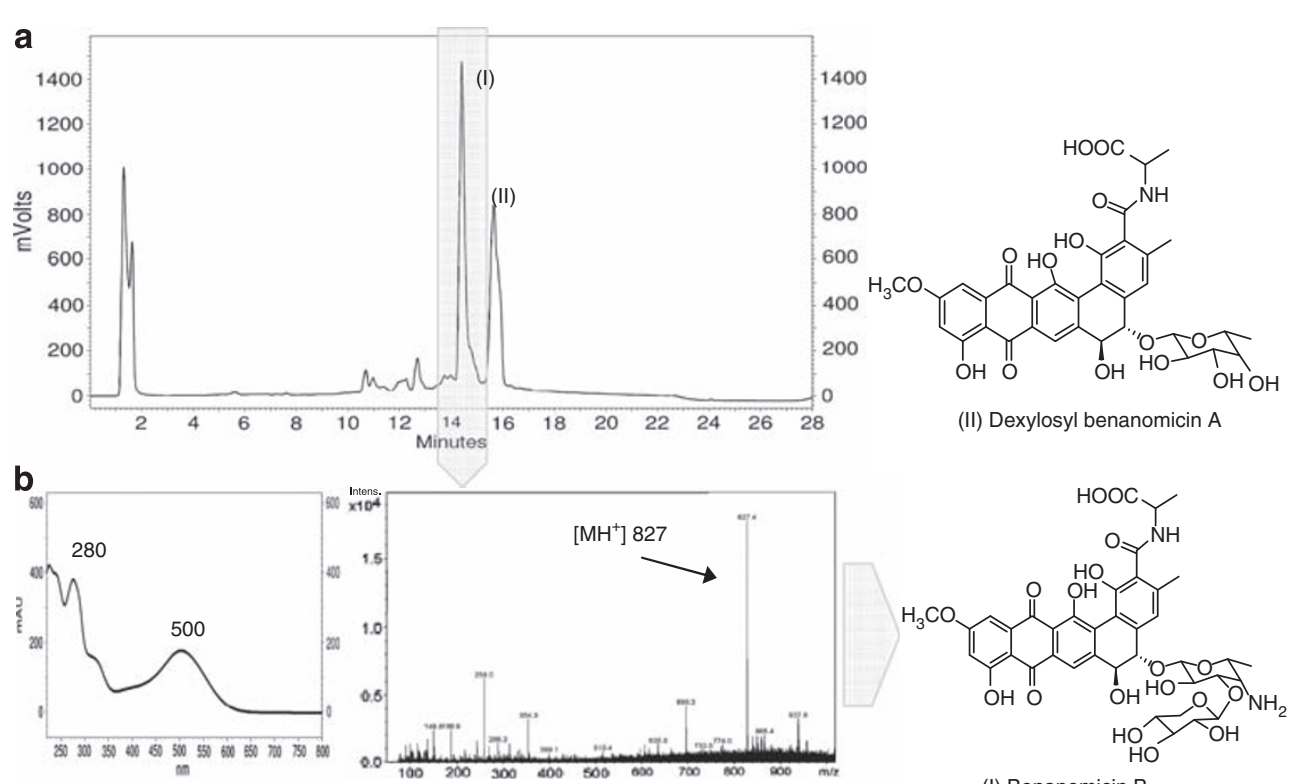

Figure 3 Identification of active metabolites from strain K15. (a) HPLC profile of methanolic extract from mycelium, with analyzed peaks labeled as (I) and (II). (b) UV-VIS and MS spectra of (I).

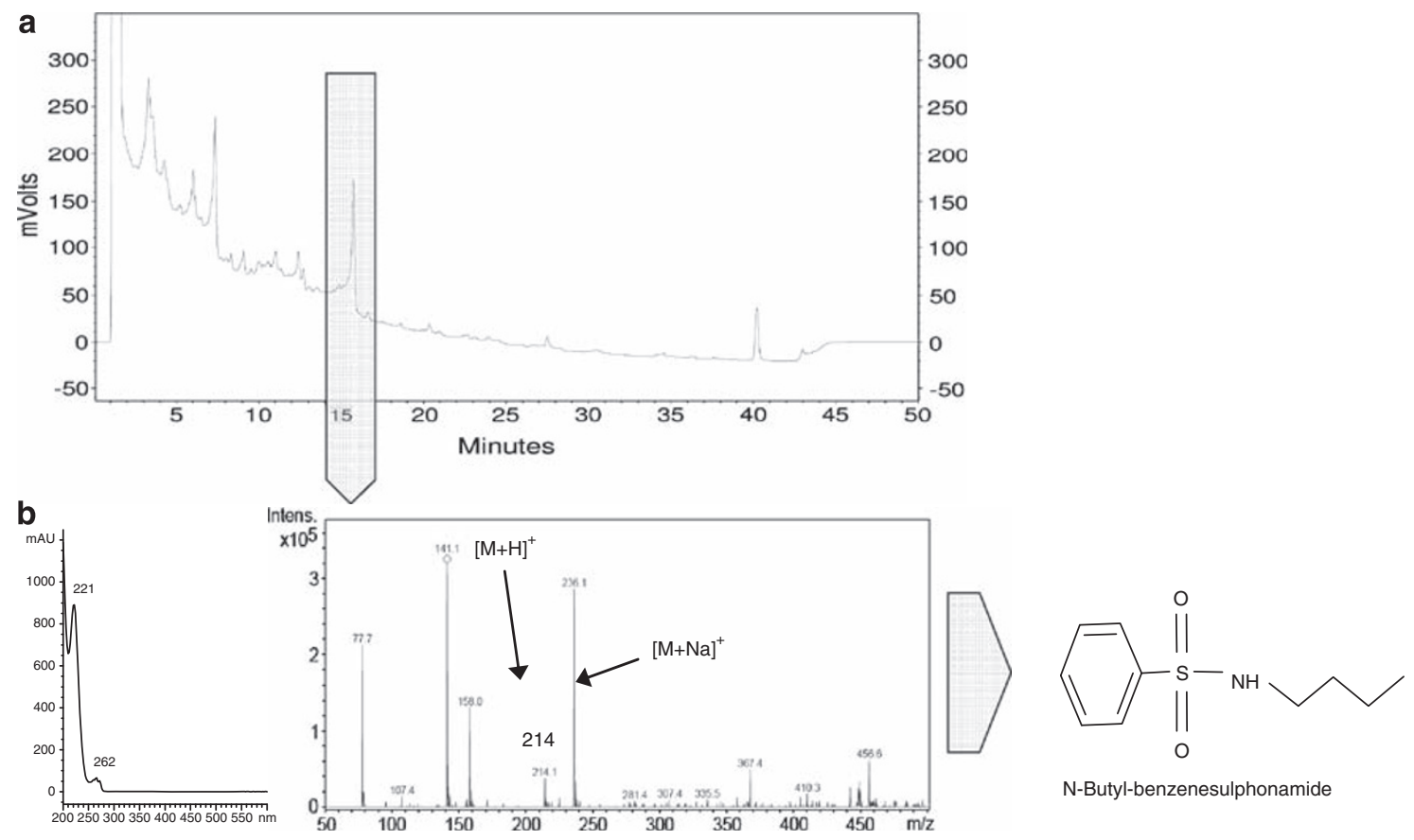

Figure 4 Identification of active metabolites from strain K216. (a) HPLC profile of ethyl acetate extract from cleared broth. (b) UV-VIS and MS spectra of (I).

Streptomyces sp. ${ }^{21}$ In addition, the Actinoallomurus K241 strain appears to be an effective producer of isoflavonoids by biotransformation of soymeal constituents.

To our knowledge, the biosynthesis of $N$-butylbenzensulphonamide has not been investigated. For the other compounds identified in this study, the benanomicin family of compounds requires the participation of a type II polyketide synthase, followed by glycosylations and other post-polyketide modifications. ${ }^{22}$ Formation of coumermycin requires the tyrosine- and proline-derived aminocoumarin and 5-methyl-pyrrole-2-carboxyl moieties, respectively, as well as a deoxysugar. ${ }^{23}$ Furthermore, we previously reported the presence of type I polyketide synthase and nonribosomal peptide synthetase genes in Actinoallomurus strains, ${ }^{7}$ a property commonly found in many Actinobacteria genera, ${ }^{24}$ including recently described lineages. ${ }^{25}$ Thus, at least some of the major pathways leading to bioactive compounds in filamentous actinomycetes are present in Actinoallomurus. These results would need to be complemented by analysis of selected genomes.

Although only four strains have been analyzed in this work, with just some new congeners from the coumermycin family identified, we 

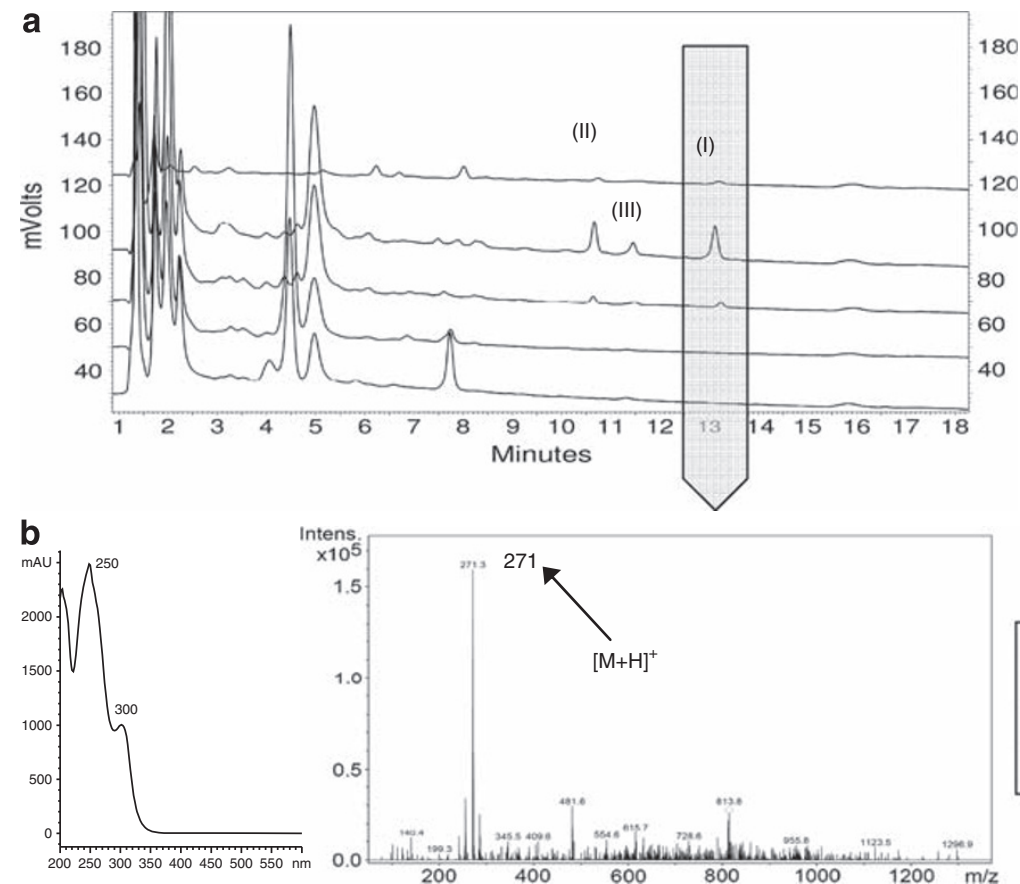<smiles>COc1cc(O)c2c(=O)c(-c3ccc(O)cc3)coc2c1</smiles>

(II) 7-O-Methyl genistein<smiles>O=c1c(-c2ccc(O)cc2)coc2cc(O)ccc12</smiles>

(III) Daidzein

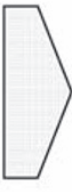<smiles>O=c1c(-c2ccc(O)cc2)coc2cc(O)cc(O)c12</smiles>

(I) Genistein

Figure 5 Metabolites produced by strain K241. (a) HPLC profiles of methanolic extracts of cleared broths from strain K241 grown in (bottom to top): RARE3, INA5-0, INA5-5 and INA5-15. Top trace is starting INA5-15. Analyzed peaks are labeled as (I), (II) and (III). (b) UV-VIS and MS spectra of (I).

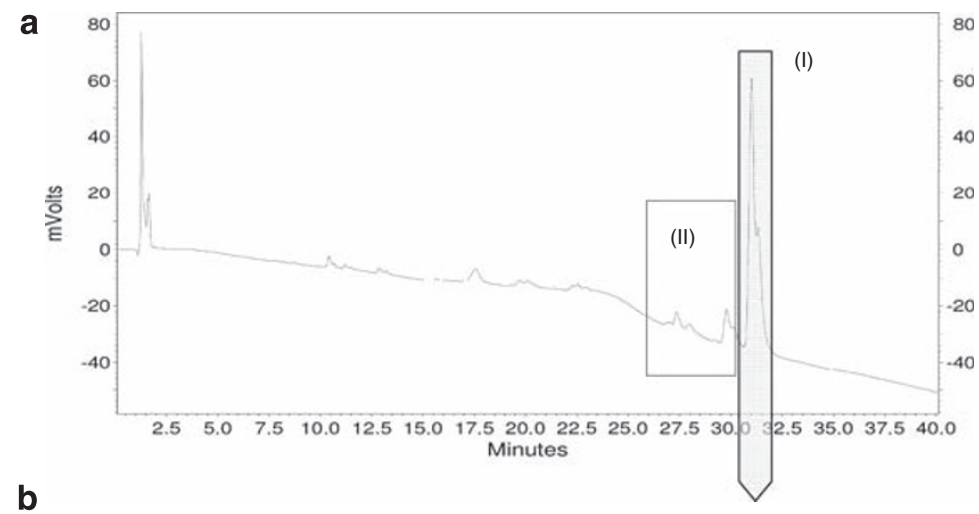

(II) Coumermycins C, D and D1
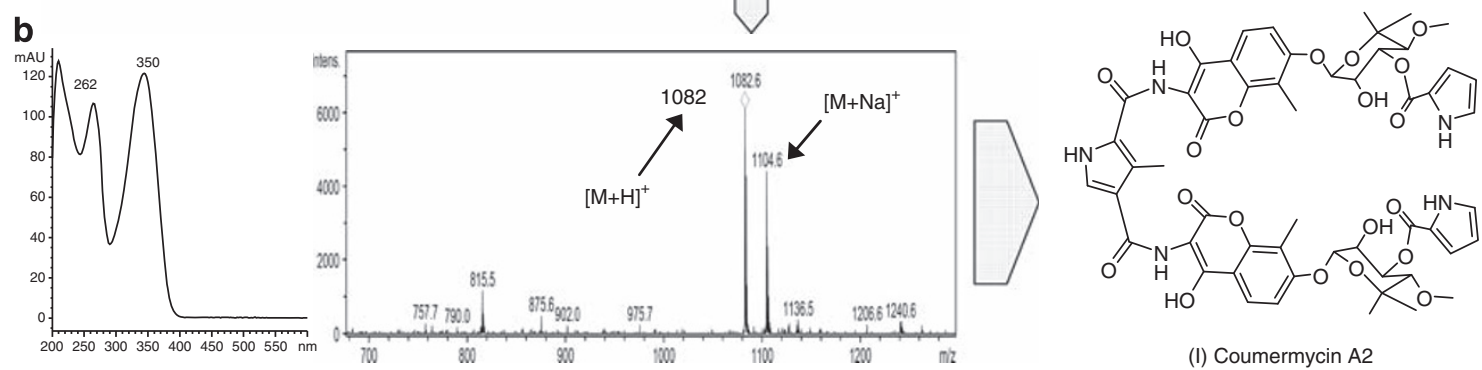

(I) Coumermycin A2

Figure 6 Identification of active metabolites from strain K275. (a) HPLC profile of methanolic extract from mycelium, with analyzed peaks labeled as (I) and (II). (b) UV-VIS and MS spectra of (I).

believe that Actinoallomurus represents a promising source of novel bioactive metabolites. In this respect, the availability of $\sim 1200$ isolates in our strain collections represents an untapped resource worth exploring.

\section{METHODS}

Bacterial strains and growth conditions

The 'Alpha' strains used in this work were derived from the KtedoGen strain collection. In some cases, their affiliation with the genus Actinoallomurus was confirmed by phylotyping. They were maintained on S15.5 plates (oatmeal $60 \mathrm{gl}^{-1}$, agar $15 \mathrm{gl}^{-1}$, adjusted to $\mathrm{pH} 5.5$ with $\mathrm{HCl}$ ). For liquid cultures, a loopful of mycelium was scraped from an agar plate, inoculated into $15 \mathrm{ml}$ of AF5.5 (see ref. 5) in a 100-ml baffled flask. For a larger scale, a 10\% inoculum from a 6-day AF5.5 culture was inoculated into $100-1000 \mathrm{ml}$ of medium. Additional media used were M8 (see ref. 5), INA5-15 (see ref. 5), V6 (see ref. 26) and RARE3 (see ref. 27). INA5-5 and INA5-0 consist of INA5-15 containing 5 and $0 \mathrm{gl}^{-1}$ soybean flour, respectively. For all media, the $\mathrm{pH}$ was 
adjusted to 5.5 with $\mathrm{HCl}$ before sterilization. All liquid cultures were incubated for $7-10$ days at $37^{\circ} \mathrm{C}$ on a rotary shaker at 200 r.p.m.

\section{PCR-selective method}

Genomic DNA was extracted with the GenElute Bacterial Genomic DNA kit (Sigma-Aldrich, Munich, Germany) or by colony picking as described. ${ }^{28}$ PCR amplification, sequence and phylogenetic analyses were performed as described. ${ }^{29}$ Strains were assigned to the same phylotype if they shared $>99.5 \%$ identity over $1400 \mathrm{bp}$ of the $16 \mathrm{~S}$ rRNA gene.

PCR primers for the specific amplification of the Actinoallomurus 16S rRNA gene were designed following a previously described procedure. ${ }^{29}$ Briefly, aligned sequences obtained from Actinoallomurus strains were compared with the consensus sequences obtained for other genera and families of the Actinomycetales, and conserved regions within the Actinoallomurus sequences were probed against the RDP database (http://rdp.cme.msu.edu/) to discard aspecific primers. The specificity of selected primer pairs was then evaluated on genomic DNAs extracted from different actinomycete genera. This procedure led to selection of primers $5^{\prime}$-CGTGAGTAACCTGCCCYYRG-3' and 5-CKTWMGCTTCGTCGGTGG-3'. Reactions were performed in a final volume of $50 \mu \mathrm{l}$, containing $50 \mathrm{~mm} \mathrm{KCl,} 10 \mathrm{~mm}$ Tris- $\mathrm{HCl}, \mathrm{pH} 8.3\left(20^{\circ} \mathrm{C}\right)$, $500 \mathrm{~nm}$ of each primer, $0.2 \mathrm{~mm}$ of each dNTP, $1.5 \mathrm{~mm} \mathrm{MgCl}_{2}, 2 \mathrm{U}$ of RedTaq DNA polymerase (Sigma-Aldrich) and $3 \mu \mathrm{l}$ of $10 \times$ Denhardt's reagent ( $2 \mathrm{~g}$ Ficoll 400, $2 \mathrm{~g}$ polyvinylpyrrolidone, $2 \mathrm{~g}$ bovine serum albumin). Amplifications consisted of 30 cycles, each comprised of $30 \mathrm{~s}$ at $94^{\circ} \mathrm{C}, 30 \mathrm{~s}$ at $60^{\circ} \mathrm{C}$ and $60 \mathrm{~s}$ at $72^{\circ} \mathrm{C}$. The program was initiated by $5 \mathrm{~min}$ at $95^{\circ} \mathrm{C}$ and terminated with $10 \mathrm{~min}$ at $72^{\circ} \mathrm{C}$. The method was validated on a panel of genomic DNAs from Actinoallomurus strains and from negative controls (strains belonging to other Actinobacteria genera, including other Thermomonosporaceae). The expected $0.34 \mathrm{kbp}$ fragment was obtained only from strains whose $16 \mathrm{~S}$ rRNA gene sequence clearly clustered within Actinoallomurus.

\section{Antimicrobial assays}

$\mathrm{MeOH}$ extracts of cultures were prepared as follows: $450 \mu \mathrm{l}$ of broth culture was shaken at 1400 r.p.m. with $900 \mu \mathrm{l} \mathrm{MeOH}$ for $1 \mathrm{~h}$ at $40^{\circ} \mathrm{C}$. The mycelium was removed by centrifugation, the supernatant was evaporated under vacuum and resuspended in $100 \mu \mathrm{l}$ of $10 \%$ DMSO. Occasionally, bioactivity was determined employing agar plugs cut from 7 to 10-day-old S15.5 plates. Agar plugs or $20 \mu \mathrm{l}$ of the resuspended $\mathrm{MeOH}$ extract were deposited on $4-\mathrm{mm}$ thick Müller Hinton Agar (Difco Laboratories, Detroit, MI, USA) plates, inoculated with $10^{5}$ c.f.u. $\mathrm{ml}^{-1}$ S. aureus ATCC 6538 P, with $10^{5}$ c.f.u. $\mathrm{ml}^{-1}$ Escherichia coli $\mathrm{K} 2526$ (a 4 tolC derivative of MG1655), or with $10^{4}$ c.f.u. $\mathrm{ml}^{-1}$ Candida albicans SKF 2270 (in the latter case, the medium was supplemented with $2 \%$ glucose). Plates were incubated overnight at $37^{\circ} \mathrm{C}$ before scoring the inhibition halos.

\section{Metabolite identification}

Metabolites were extracted from liquid cultures by adding two volumes of $\mathrm{MeOH}$ to the entire culture and thoroughly mixing for $1 \mathrm{~h}$ at $50^{\circ} \mathrm{C}$. After centrifugation, the liquid phase was dried under vacuum and dissolved in $0.5 \mathrm{ml} \mathrm{10 \%} \mathrm{DMSO} \mathrm{per} \mathrm{ml}$ of original culture. An aliquot was fractionated on a Shimadzu Series 10 system (Kyoto, Japan), equipped with a reversed-phase column, Lichrosorb RP-18, $5 \mu \mathrm{m}, 4 \times 250 \mathrm{~mm}$ (Merck, Darmstadt, Germany) and a diode array detector, using a linear gradient of $0.05 \mathrm{M}$ ammonium formate-acetonitrile (from 5 to $90 \%$ of organic phase in $30 \mathrm{~min}$ ) at a flow rate of $1 \mathrm{ml} \mathrm{min}{ }^{-1}$. Fractions $(1 \mathrm{ml})$ from the HPLC column were collected, dried and resuspended in $100 \mu \mathrm{l} 10 \%$ DMSO, and $20 \mu \mathrm{l}$ was tested for bioactivity.

Preparative HPLC employed a Shimadzu Series 10 spectrophotometer, Merck Lichrosphere C18 4.6×100-mm column, diode array detector (190$800 \mathrm{~nm})$, using a linear gradient of eluents A $\left(0.1 \%\right.$ TFA in $\left.0.05 \mathrm{M} \mathrm{HCOONH}_{4}\right)$ and B (acetonitrile) from 5 to $90 \% \mathrm{~B}$ in $30 \mathrm{~min}$ at a flow rate of $1 \mathrm{ml} \mathrm{min}^{-1}$. Reversed-phase medium pressure liquid chromatography was performed on a Combiflash ISCO (Teledyne ISCO, Lincoln, NE, USA) RP18 $12 \mathrm{~g}$ column, with a 20 -min linear gradient from 5 to $90 \%$ eluent B in $20 \mathrm{~min}$ at $18 \mathrm{ml} \mathrm{min}^{-1}$. ESI-MS data were recorded on a MS Bruker (Karlsruhe, Germany) Ion Trap ESQUIRE 3000 Plus spectrometer, LC Agilent 1100 DAD. ${ }^{1} \mathrm{H}$ and $1{ }^{3} \mathrm{C} 1-$ and
2D-NMR spectra (COSY, TOCSY, NOESY, HSQC, HMBC) were measured in DMSO-d6, $\mathrm{CDCl} 3$ or MeOD-d4, on Bruker 400 or $600 \mathrm{MHz}$ instruments.

The UV properties and mass data of the molecules present in the active fractions were queried against the ABL database, which contains data on approximately 29000 microbial metabolites published up to 2006. An earlier version of the database was previously described. ${ }^{30}$

\section{ACKNOWLEDGEMENTS}

Work at KtedoGen was partially supported by grants from Italian Ministry of Research and from Regione Lombardia. M Simone and C Mazzetti were directly supported from a fellowship from Regione Lombardia.

1 Fischbach, M. A. \& Walsh, C. T. Antibiotics for emerging pathogens. Science 325, 1089-1093 (2009).

2 Donadio, S., Maffioli, S., Monciardini, P., Sosio, M. \& Jabes, D. Antibiotic discovery in the twenty-first century: current trends and future perspectives. J. Antibiot. 63, 423-4530 (2010).

3 Maier, A. et al. Biomolecular-chemical screening: a novel screening approach for the discovery of biologically active secondary metabolites. I. Screening strategy and validation. J. Antibiot. 52, 945-951 (1999).

4 Corre, C. \& Challis, G. L. New natural product biosynthetic chemistry discovered by genome mining. Nat Prod Rep. 26, 977-986 (2009).

5 Donadio, S., Monciardini, P. \& Sosio, M. Approaches to discovering novel antibacterial and antifungal agents. Meth. Enzymol. 438, 3-28 (2009).

6 Singh, S. B. \& Young, K. New antibiotic structures from fermentations. Expert Opin. Ther. Pat. 20, 1359-1371 (2010).

7 Donadio, S. et al. Sources of polyketides and non-ribosomal peptides. Ernst Schering Res Found Workshop 51, 19-41 (2005).

8 Busti, E. et al. Catenulispora acidiphila gen. nov., sp. nov., a novel mycelium-forming actinomycete and proposal of Catenulisporaceae fam. nov.. Int. J. Syst. Evol. Microbiol. 56, 1741-1756 (2006).

9 Cavaletti, L. et al. Actinospica acidiphila gen. nov., sp. nov., and Actinospica robiniae gen. nov., sp. nov.; proposal for Actinospicaceae fam. nov. and Catenulisporinae subordo nov. in the order Actinomycetales. Int. J. Syst. Evol. Microbiol. 56, 1747-1753 (2006).

10 Monciardini, P. et al. Two novel members of the family Micromonosporaceae, Rugosimonospora acidiphila gen. nov, sp. nov. and Rugosimonospora africana sp. nov. Int. J. Syst. Evol. Microbiol. 59, 2752-2758 (2009).

11 Tamura, T, Ishida, Y Nozawa, Y, Otoguro, M. \& Suzuki, K. Transfer of Actinomadura spadix Nonomura and Ohara 1971 to Actinoallomurus spadix gen. nov., comb. nov., and description of Actinoallomurus amamiensis sp. nov., Actinoallomurus caesius sp. nov., Actinoallomurus coprocola sp. nov., Actinoallomurus fulvus sp. nov., Actinoallomurus iriomotensis sp. nov., Actinoallomurus luridus sp. nov., Actinoallomurus purpureus sp. nov. and Actinoallomurus yoronensis sp. nov. Int. J. Syst. Evol. Microbiol. 59, 1867-1874 (2009).

12 Gomi, S. et al. The structures of new antifungal antibiotics, benanomicins A and B. J. Antibiot. 41, 1019-1028 (1988).

13 Kim, K. K., Kang, J. G., Moon, S. S. \& Kang, K. Y. Isolation and identification of antifungal $\mathrm{N}$-butylbenzenesulphonamide produced by Pseudomonas sp. AB2. J. Antibiot. 53, 131-136 (2000).

14 Dixon, R. A. \& Ferreira, D. Genistein. Phytochemistry 60, 205-211 (2002).

15 Hazato, T., Naganawa, H., Kumagai, M., Aoyagi, T. \& Umezawa, H. beta-Galactosidaseinhibiting new isoflavonoids produced by actinomycetes. J. Antibiot. 32, 217-222 (1979).

16 Anyanwutaku, I. O., Zirbes, E. \& Rosazza, J. P. N. Isoflavonoids from streptomycetes: origins of genistein, 8-chlorogenistein, and 6,8-dichlorogenistein. J. Nat. Prod. 55, 1498-1504 (1992).

17 Kawaguchi, H. et al. Studies on coumermycin, a new antibiotic-Production, isolation and characterization of coumermycin A1. J. Antibiot. 8, 11-25 (1964).

18 Janso, J. E. \& Carter, G. T. Biosynthetic potential of phylogenetically unique endophytic actinomycetes from tropical plants. Appl. Environ. Microbiol. 76, 4377-4386 (2010).

19 Thamchaipenet, A. et al. Actinoallomurus acaciae sp. nov., an endophytic actinomycete isolated from Acacia auriculiformis A. Cunn. ex Benth. Int. J. Syst. Evol. Microbiol. 60, 554-559 (2010).

20 Indananda, C., Thamchaipenet, A., Matsumoto, A., Duangmal, K. \& Takahashi, Y. Actinoallomurus oryzae sp. nov., an endophytic actinomycete isolated from root of Thai jasmine rice plant. Int. J. Syst. Evol. Microbiol. (doi:10.1099/ijs.0.022509-0) (2010).

21 Elleuch, L. et al. Bioactive secondary metabolites from a new terrestrial Streptomyces sp. TN262. Appl. Biochem. Biotechnol. 162, 579-593 (2010).

22 Zhan, J. Biosynthesis of bacterial aromatic polyketides. Curr. Top. Med. Chem. 9, 1598-1610 (2009).

23 Heide, L. The aminocoumarins: biosynthesis and biology. Nat. Prod. Rep. 26, 1241-1250 (2009).

24 Donadio, S., Monciardini, P. \& Sosio, M. Polyketide synthases and nonribosomal peptide synthetases: the emerging view from bacterial genomes. Nat. Prod. Rep. 24, 1073-1109 (2007). 
25 Busti, E. et al. Antibiotic producing ability by representatives of a newly discovered lineage of actinomycetes. Microbiology 152, 675-683 (2006).

26 Binz, T. M., Maffioli, S. I., Sosio, M., Donadio, S. \& Müller, M. Insights into an unusual nonribosomal peptide synthetase biosynthesis: identification and characterization of the GE81112 biosynthetic gene cluster. J. Biol. Chem. 285, 32710-32719 (2010).

27 Sosio, M., Stinchi, S., Beltrametti, F., Lazzarini, A. \& Donadio, S. The gene cluster for the biosynthesis of the glycopeptide antibiotic A40926 by Nonomuraea species. Chem. Biol. 10, 541-549 (2003).
28 Mazza, P., Monciardini, P., Cavaletti, L., Sosio, M. \& Donadio, S. Diversity of Actinoplanes and related genera isolated from an Italian soil. Microb. Ecol. 45, 362-372 (2003).

29 Monciardini, P., Sosio, M., Cavaletti, L., Chiocchini, C. \& Donadio, S. New primers for the selective amplification of 16S rDNA from different groups of actinomycetes. FEMS Microbiol. Ecol. 42, 419-429 (2002).

30 Lazzarini, A., Cavaletti, L., Toppo, G. \& Marinelli, F. Rare genera of actinomycetes as potential producers of new antibiotics. Antonie Van Leeuwenhoek 79, 399-405 (2000). 